DOI: https://doi.org/10.46296/yc.v4i7.0042

\title{
PROPUESTA DE MANEJO DE RESIDUOS SÓLIDOS EN LA FACULTAD DE CIENCIAS MATEMÁTICAS, FÍSICAS Y QUÍMICAS. UTM.
}

\section{SOLID WASTE MANAGEMENT PROPOSAL IN THE FACULTY OF MATHEMATICAL, PHYSICAL AND CHEMICAL SCIENCES. UTM.}

\author{
Cedeño-Guillen Angelo Divaldo ${ }^{1 *}$; Perero-Espinoza Galo Arturo² \\ 1 Universidad Técnica de Manabí (UTM) - Instituto de Posgrado. Portoviejo, Ecuador. \\ ${ }^{2}$ Docente Investigador de la Universidad Técnica de Manabí (UTM). Portoviejo, Ecuador.
}

*Correo: galo.perero@utm.edu.ec

\section{Resumen}

El principal productor de desechos sólidos (orgánicos e inorgánicos) es el hombre. En este contexto se hace necesario una visión de responsabilidad social e institucional en el tema del manejo de estos, porque la temática es de interés mundial (Vergara Villamizar \& Castillo Payares, 2020). Las instituciones están llamadas a promover la concientización y manejo de dichos residuos. El trabajo de investigación fue realizado en la Facultad de Ciencias Matemáticas, Físicas y Químicas de la Universidad Técnica de Manabí y estableció como objetivo, elaborar una propuesta para la gestión del manejo de residuos sólidos basado en la norma ISO 14001:2015, con la finalidad de realizar una clasificación y recolección de datos del manejo de residuos sólidos orgánicos (residuos de comida) e inorgánicos (plásticos, papeles, vidrios, metales) que genera el área de estudio. La norma ISO utilizada presentó cuatro etapas siguiendo los lineamientos estándar: planificación, operación, evaluación y la actuación, esta es necesaria por su intención de propiciar la mejora continua en la calidad ambiental de su tratamiento y aumentar el desempeño del sistema de gestión ambiental dentro de la Facultad. Se utilizaron como instrumentos, técnicas de recolección de datos tales como: la observación de campo, encuestas y entrevistas que fueron realizadas a quienes forman parte de la facultad. Finalmente, se comprobó, que la problemática fue ocasionada por la falta de conciencia y poca cultura ecológica por parte de una fracción importante de estudiantes, docentes y personal administrativo. Al ser identificados los residuos que se generan y sus causas, se pudo concluir que la ejecución de la propuesta es de vital importancia para la facultad de matemáticas, puesto que esta se encuentra inmersa actualmente en una problemática ecológica, la cual afecta directamente al medio ambiente y a las labores diarias de dicha comunidad universitaria.

Palabras claves: residuos, orgánicos, inorgánicos, norma ISO 14001:2015, sistemas de gestión.

\begin{abstract}
The main producer of solid waste (organic and inorganic) is man. In this context, a vision of social and institutional responsibility in the management of these wastes is necessary, because the subject is of global interest (Vergara Villamizar \& Castillo Payares, 2020). Institutions are called upon to promote awareness and management of such waste. The research work was carried out at the Faculty of Mathematics, Physics and Chemistry of the Technical University of Manabí and established the objective of developing a proposal for solid waste management based on ISO 14001:2015, in order to classify and collect data on the management of organic (food waste) and inorganic (plastics, paper, glass, metals) solid waste generated by the area of study. The ISO standard used presented four stages following the standard guidelines: planning, operation, evaluation and performance, this is necessary for its intention to promote continuous improvement in the environmental quality of their treatment and increase the performance of the environmental management system within the Faculty. Data collection techniques were used as instruments, such as: field observation, surveys and interviews that were carried out with those
\end{abstract}

Información del manuscrito:

Fecha de recepción: 24 de abril de 2020

Fecha de aceptación: 09 de julio de 2020

Fecha de publicación: 10 de julio de 2020 
who are part of the faculty. Finally, it was proved that the problem was caused by the lack of awareness and little ecological culture on the part of an important fraction of students, teachers and administrative staff. Once the waste generated and its causes were identified, it was concluded that the implementation of the proposal is of vital importance for the mathematics faculty, since it is currently immersed in an ecological problem, which directly affects the environment and the daily work of the university community.

Keywords: waste, organic, inorganic, ISO 14001:2015, management systems.

\section{Introducción}

La protección del medio ambiente se reconoce cada vez más como un factor clave en la salud pública. Las instituciones de educación superior no solo juegan un papel en la investigación y la docencia, sino que también sirven como espacios de convivencia y desarrollo de hábitos y actitudes, se han realizado diversas acciones para la búsqueda de sólidos sostenibles en los campos de la energía, el transporte, el agua y la gestión de residuos. Estos comportamientos no solo tienen un impacto en la gestión ambiental de la organización, sino que también tienen un impacto en la educación, porque la visión y experiencia de los estudiantes sobre las prácticas ambientales afectará su formación. (Ruiz Morales, 2017)

Según (Mejía Miranda \& Patarón Andino, 2014) un desecho sólido es todo sólido putrescible 0 no putrescible, excepto excretas de origen humano o animal, como, por ejemplo: los desperdicios de hogares, industrial, hospitales no contaminantes, plazas de mercados, escombros, entre otros (p. 24).

Mientras que los residuos sólidos como aquellas sustancias, productos o subproductos en estado sólido o semisólido de los que su generador dispone, o está obligado a disponer, en virtud de lo establecido en la Normatividad Nacional o de los riesgos que causan a la salud y el ambiente, para ser manejados a través de un sistema que incluya, según corresponda. (Herrera Fuentes, 2015)

Los residuos sólidos, constituyen aquellos materiales desechados tras su vida útil, y que por lo general por sí solos carecen de valor económico, se componen principalmente de desechos procedentes de materiales utilizados en la fabricación, transformación o utilización de bienes de consumo. Todos estos residuos sólidos, en su mayoría son susceptibles de reaprovecharse 0 
transformarse con un correcto reciclado. Los principales "productores" de residuos sólidos son los ciudadanos de las grandes ciudades, con un porcentaje muy elevado, en especial por la poca conciencia del reciclaje que existe en la actualidad. Afortunadamente esto está cambiando poco a poco, pero problemas como el cambio climático, son ahora una amenaza real y a corto plazo. (Residuos sólidos. Definición, s. f.)

El manejo de residuos sólidos puede cambia la forma de vivir de muchas personas, puesto que si su tratamiento es inadecuado puede producir diversos inconvenientes al medio al cual son arrojados, Cada actividad que se realiza día a día produce residuos sólidos, estos tienen unas etapas de ciclo de vida entre las cuales podemos distinguir las siguientes: generación, transportación, almacenamiento, recolección, tratamiento y disposición final, las organizaciones o instituciones contribuyen a este escenario en el cual se despliegan diferentes tipos de actividades que producen estos residuos.

El propósito de la norma ISO 14001:2015 es proporcionar un marco de referencia para que las organizaciones protejan el medio ambiente y respondan a las condiciones ambientales cambiantes, teniendo en cuenta las necesidades socioeconómicas. El estándar especifica los requisitos que permiten a las organizaciones lograr los resultados esperados que ha logrado su sistema de gestión ambiental. (ISO 14001:2015(es), s. f.)

Un punto clave que ayuda a la propuesta es la norma ISO 14001:2015 la misma que es una herramienta importante que proporciona un marco para el manejo eficaz de los residuos que pueden generar las instituciones, si no es para disminuir la cantidad de residuos, por lo menos busca manejarlos de forma correcta.

El presente trabajo se llevó a cabo con el propósito de elaborar una propuesta que puedan ayudar a manejar de forma correcta los desechos sólidos que son generados en la Facultad de Ciencias Matemáticas, Físicas y Químicas de la Universidad Técnica de Manabí. 


\section{Metodología}

Según (Grass Lozano, 2017) la elaboración de una investigación empieza por la etapa de diagnóstico, que consiste en un profundo análisis de la situación de la comunidad y de las problemáticas que le impiden su correcto desarrollo (p.17).

El desarrollo de la propuesta de manejo de residuos sólidos en la facultad de la Universidad Técnica de Manabí busca generar una guía con lineamientos para el mejoramiento de la gestión ambiental en el lugar, se emplea la norma ISO 14001: 2015 porque permitió la identificación de los problemas de la institución que afectan al ambiente, la generación de objetivos de mejora y un programa de gestión para alcanzarlos, esto ayuda a aportar tanto en el ámbito social, académico y económico a la facultad.

Esta investigación se llevó a cabo mediante investigación de campo, donde se realizó encuestas, entrevistas y observación de campo, también por medio bibliográfico documental debido a que se llevó acabo por recolección de fuentes secundarias para fundamentar los principales temas de la investigación.

El método aplicado es HIPOTÉTICO-DEDUCTIVO, puesto que se plantea hipótesis que se pueden analizar deductiva y/o inductivamente, con su correspondiente comprobación experimental, es decir, se busca que la parte teórica no pierda su sentido, por ello se relaciona posteriormente con la realidad. Es preciso recordar que una de las características de este método es la combinación de otros métodos así: el inductivo, el deductivo y el experimental.

\section{Resultados y discusión}

La población investigada fueron los estudiantes, docentes, empleados de servicio y administrativos que desempeñan sus actividades en la Facultad de Ciencias Matemáticas, Físicas y Químicas de la Universidad Técnica de Manabí constituido por 4097 personas compuesto de la siguiente forma:

- 3968 estudiantes.

- 114 docentes.

- 8 del personal administrativo.

- 7 operadores de servicio.

El tamaño de la muestra se obtiene a partir de la fórmula que se muestra 
a continuación, y que además determina el número de personas a encuestar.

$\mathrm{N}=4097$

$p=0,5$

$\mathbf{q}=0,5$

$\mathbf{E}=0,06$

$Z=94 \%=1,88$

$n=\frac{4097(1,88)^{2}(0,5)(0,5)}{(0,06)^{2}(4097-1)+(1,88)^{2}(0,5)(0,5)}=232$

Para la aplicación del cuestionario estructurado se procedió a encuestar a 232 personas que según el tipo de miembro de la comunidad académica se escogió la siguiente muestra:

Tabla 1. Distribución del tamaño de la muestra.

\begin{tabular}{|c|c|c|c|}
\hline Muestra & $\begin{array}{c}\text { Total, de } \\
\text { Universo }\end{array}$ & $\begin{array}{c}\text { Relación } \\
\text { porcentual }\end{array}$ & $\begin{array}{c}\text { Tamaño } \\
\text { de la } \\
\text { muestra }\end{array}$ \\
\hline Docentes & 114 & 0,0278 & 6 \\
\hline Administrativos & 8 & 0,0020 & 2 \\
\hline Estudiantes & 3968 & 0,9685 & 222 \\
\hline Servicio & 7 & 0,0017 & 2 \\
\hline TOTAL & $\mathbf{4 0 9 7}$ & $\mathbf{1 , 0 0 0 0}$ & $\mathbf{2 3 2}$ \\
\hline
\end{tabular}

Fuente: Elaboración propia.

Por lo tanto, la composición de la muestra se definió con 6 docentes, 2 trabajadores de oficinas, 222 estudiantes, y 2 operadores de servicio.
Gráfico 1. Encuesta realizada para obtener muestras de la investigación ejecutada.

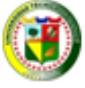

UNIVERSIDAD TECNICA DE MANAB INSTITUTO DE POSGRADO

MAESTRIA EN GERENCIA DE LA CALIDAD E INNOVACION

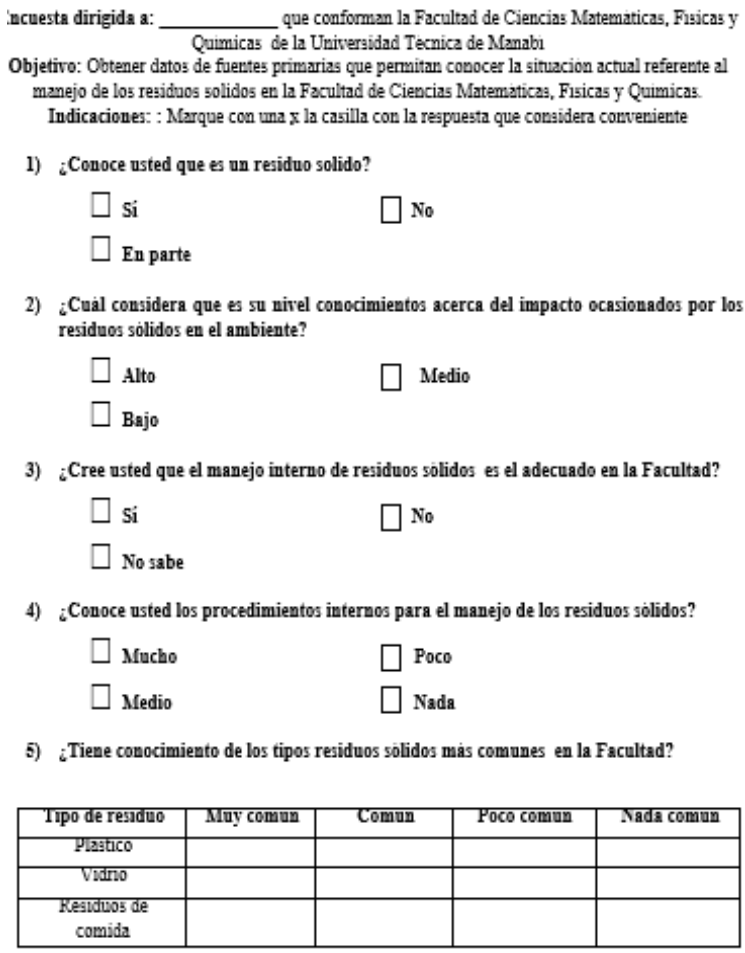

Fuente: Elaboración propia.

En la actualidad en la Facultad de Ciencias Matemáticas, Físicas y Químicas de la Universidad Técnica de Manabí se constató que existe una leve problemática del manejo inadecuado de residuos sólidos ocasionado por el desconocimiento de una parte considerable de la población de estudio en relación con la debida gestión e información relacionada. 
Gráfico 2. Conocimiento sobre lo que es un residuo sólido.

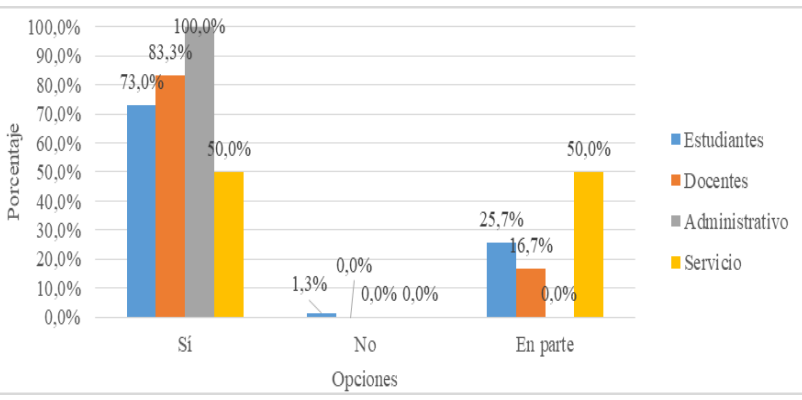

Fuente: Encuesta directa a miembros de la Facultad de Ciencias Matemáticas Físicas y Químicas. Elaboración propia.

Gráfico 3. Nivel de conocimientos acerca del impacto ocasionado por los residuos sólidos en el ambiente.

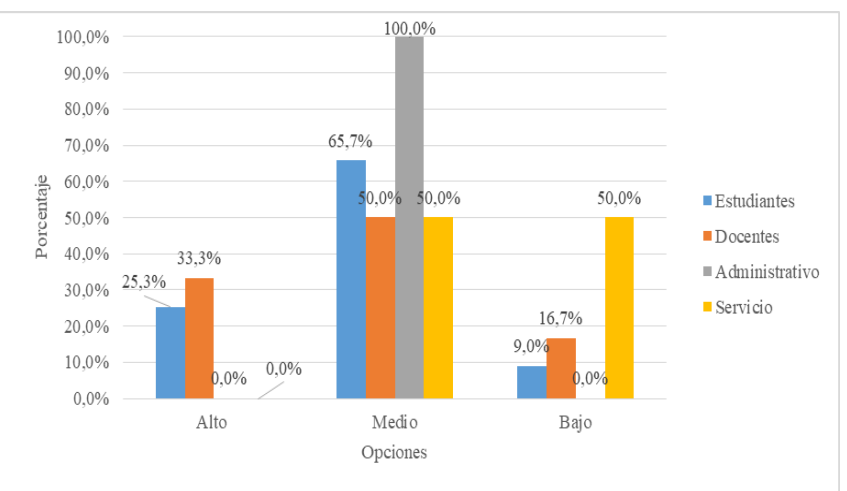

Fuente: Encuesta directa a miembros de la Facultad de Ciencias Matemáticas Físicas y Químicas. Elaboración propia.

Gráfico 4. Sobre si el manejo interno de residuos sólidos es el adecuado en la Facultad.

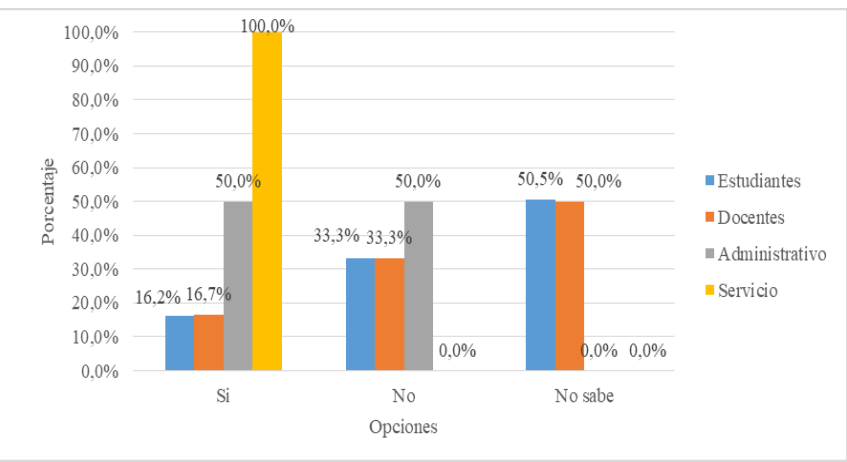

Fuente: Encuesta directa a miembros de la Facultad de Ciencias Matemáticas Físicas y Químicas. Elaboración propia.
Gráfico 5. Conocimiento sobre los procedimientos internos para el manejo de los residuos y desechos solidos

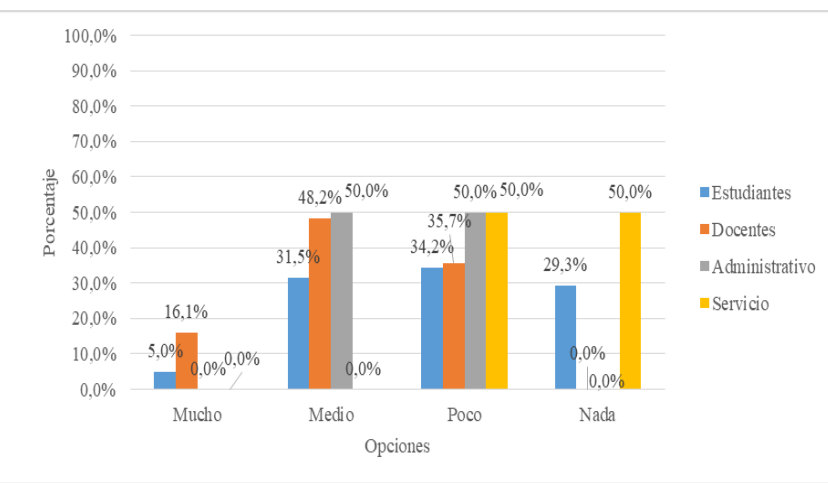

Fuente: Encuesta directa a miembros de la Facultad de Ciencias Matemáticas Físicas y Químicas. Elaboración propia.

Gráfico 6. Tipos de residuos sólidos más comunes que se generan en la Facultad.

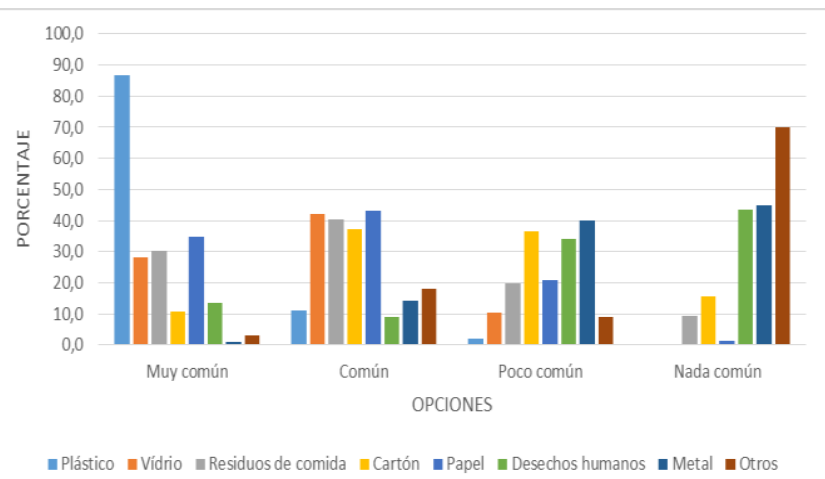

Fuente: Encuesta directa a miembros de la Facultad de Ciencias Matemáticas Físicas y Químicas. Elaboración propia.

Este objetivo se cumplió mediante la aplicación de encuestas y la observación de campo pudiendo identificar que el $25,4 \%$ de los miembros que conforman la Facultad tienen conocimientos parciales sobre lo que son los residuos sólidos pudiendo incluso llegar a tener inconvenientes para distinguirlos y separarlos de forma correcta, el 
$65,7 \%$ de estos mencionó tener un nivel medio acerca del conocimiento del impacto que puede ocasionar estos residuos en el ambiente, el $50.5 \%$ desconoce totalmente los procedimientos interno para el manejo de los residuos en la facultad, el $34.2 \%$ tiene un nivel de conocimiento bajo sobre los procedimientos de los residuos, el $28,0 \%$ conoce poco acerca de los tipos de residuos sólidos de cada residuo solidos según su naturaleza.

\section{Conclusiones}

Se analizó la situación actual del manejo de residuos sólidos que atraviesa la Facultad de Ciencias Matemáticas, Físicas y Químicas identificando que existe una leve problemática de un manejo inadecuado de residuos ocasionado por la falta de conciencia y poca cultura ecológica por parte de un porcentaje de la población, en su mayoría estudiantes, determinando que no solo se debe a la falta de información sino también al desinterés existente en el cuidado del ambiente.

Los elementos relacionados al manejo de residuos sólidos basados en la norma ISO 14001: 2015, establece que la gestión de residuos sólidos puede definirse como las técnicas que garantizarán las ejecuciones respectivas de las actividades y funciones relacionadas a la recolección, transporte, procesamiento, tratamiento $\mathrm{y}$ eliminación de esto.

La propuesta realizada se basó en un diseño de manejo de residuos sólidos utilizando la norma ISO 14001: 2015, y en la que se aplicaron los requisitos y clausulas sugeridas en el estándar. Se basó principalmente en las etapas de planificación, operación, evaluación y mejorado del ambiente.

\section{Bibliografía}

Grass Lozano, L. S. (2017). Guía para la implementación de la metodología para la elaboración del plan de gestión integral de residuos sólidos en COLINAGRO S. A. 25.

Herrera Fuentes, E. A. (2015). Aplicación de la ley general de residuos sólidos y sus efectos en la calidad de vida de la población de Chancay 2014. 24. 
ISO 14001:2015(es).

(s. f.).

Bienvenido a la plataforma de navegación en línea (OBP). Recuperado 23 de septiembre de 2020, de https://www.iso.org/obp/ui/\#is o:std:iso:14001:ed-3:v1:es

Mejía Miranda, P. A. M., \& Patarón Andino, I. M. P. (2014). Ingenieras En Biotecnología Ambiental. 262.

Residuos sólidos. Definición. (s. f.). Recuperado 19 de septiembre de 2020, de https://www.inforeciclaje.com/ residuos-solidos.php

Ruiz Morales, M. (2017). Contexto y evolución del plan de manejo integral de residuos sólidos en la

Universidad Iberoamericana ciudad de México. Revista Internacional de Contaminación Ambiental, 33(2), 337-346. https://doi.org/10.20937/RICA .2017.33.02.14

Vergara Villamizar, M. A., \& Castillo Payares, R. (2020). Responsabilidad social ambiental en el manejo de residuos sólidos en los fabricantes de calzados en el municipio de Chinú Córdoba.

https://repositorio.unicordoba. edu.co/handle/ucordoba/3012 